\title{
Late childhood interpersonal callousness and conduct problem trajectories interact to predict adult psychopathy
}

\author{
Samuel W. Hawes, ${ }^{1}$ Amy L. Byrd, ${ }^{2}$ Rebecca Waller, ${ }^{3}$ Donald R. Lynam, ${ }^{4}$ and \\ Dustin A. Pardini ${ }^{1}$ \\ ${ }^{1}$ School of Criminology and Criminal Justice, Arizona State University, Phoenix, AZ; ${ }^{2}$ Department of Psychiatry, \\ University of Pittsburgh, Pittsburgh, PA; ${ }^{3}$ Department of Psychology, University of Michigan, Ann Arbor, MI; \\ ${ }^{4}$ Department of Psychological Sciences, Purdue University, West Lafayette, IN, USA
}

\begin{abstract}
Background: Studies have demonstrated a robust association between interpersonal callousness (IC) and the development of severe and chronic conduct problems (CP) in youth. Although children exhibiting IC are also believed to be at particularly high risk for developing psychopathic personality features in adulthood, there is little longitudinal evidence supporting this assumption, particularly after controlling for co-occuring CP severity. Methods: This study used data collected on a longitudinal cohort of boys $(n=508)$, with an oversampling of youth exhibiting elevated conduct problems. Analyses examined the unique and interactive association between latent growth curve trajectories of IC and CP assessed bi-annually from late childhood to early adolescence ( ages 10-13) and features of psychopathy in early adulthood (age 24) assessed using the Psychopathy Checklist - Short Version (PCL:SV; Hart, Cox, \& Hare, 1995). Results: Growth curve analysis indicated that initial levels of IC and CP in childhood ( age 10 intercept) both uniquely predicted the development of the interpersonal/affective features of adult psychopathy, and boys with a combination of high initial levels of IC and CP were at particularly high risk for developing the impulsive/antisocial features of the disorder. Boys who exhibited systematic increases in CP from late childhood to early adolescence also demonstrated higher adult psychopathy scores, but changes in IC across this developmental period did not significantly add to the prediction of adult psychopathy. Conclusions: Findings highlight the importance of developing targeted interventions for boys exhibiting severe IC and CP in childhood, as they appear to be at high risk for developing adult psychopathic features. Keywords: Interpersonal callousness; conduct problems; psychopathy; trajectories.
\end{abstract}

\section{Introduction}

Psychopathy is a complex disorder, characterized by a distinct constellation of interpersonal, affective, and behavioral features. Due to the disproportionate amount of violence and other criminal behaviors associated with psychopathy (Patrick, 2007), research has focused on its early identification, developmental precursors, and the relationships of its underlying facets. In particular, several authors have focused on delineating the interpersonal (e.g. superficial charm, manipulative, deceitful behavior) and affective (e.g. callousness, lack of remorse) features of psychopathy in youth, which will hereafter be referred to as interpersonal callousness (IC). ${ }^{1}$ Over the past several decades, longitudinal studies have found that children and adolescents with high levels of IC are at heightened risk for exhibiting a severe and chronic pattern of violence and other criminal behavior. However, few studies have examined the extent to which features of IC and early conduct problems (e.g. physical aggression, theft, destruction of property) unfold in tandem from childhood to adolescence, and whether youth with

Conflict of interest statement: No conflicts declared. high IC will go on to exhibit psychopathic personality characteristics into young adulthood.

It is commonly assumed that IC emerges in early childhood and subsequently drives the development of aggression and other severe behavior problems. In essence, antisocial behaviors are posited to occur developmentally downstream from the emergence of the interpersonal and affective features of psychopathy (Pardini, Waller, \& Hawes, 2015; Skeem \& Cooke, 2010). However, early engagement in conduct problems (CP) and involvement in a deviant lifestyle could also shape the development of these features. For example, adolescents often engage in delinquent behavior as part of a larger deviant peer group, and longitudinal evidence suggests that as adolescents spend more time with delinquent peers, their tendency to view victimizing others as morally acceptable behavior increases (e.g. Pardini, Loeber, \& Stouthamer-Loeber, 2005). It is also possible that IC and CP unfold in tandem across development because they are being driven by common causal factors. Consistent with this notion, a hostile parenting style and parental conflict in the home have been linked to increased engagement in early $\mathrm{CP}$ and may lessen a child's ability to internalize prosocial norms and values (for a review, see Pardini et al., 
2015; Waller, Gardner, \& Hyde, 2013). In sum, although longitudinal studies suggest that IC features exhibt moderate stability during childhood and adolescence (Hawes, Mulvey, Schubert, \& Pardini, 2014; Hyde et al., 2013), the extent to which changes in IC and CP cooccur during this developmental period remains unclear.

Another key issue that has not been sufficiently addressed is the extent to which early features of IC represent a developmental precursor to adult psychopathy. Achieving a clearer understanding of the link between early IC and adult psychopathy is important for two reasons. First, it would help to validate the notion that early precursors of the interpersonal and affective features of adult psychopathy can be assessed during childhood (Seagrave \& Grisso, 2002; p. 233). Second, since adult offenders with psychopathic features are at high risk for violently re-offending (Patrick, 2007) and have proven difficult to engage in treatment (Rice $\&$ Harris, 1997), studies in this area are useful for determining whether early preventive-interventions should explicitly target youth exhibiting features of IC. Relatedly several studies have found that existing evidence-based treatments are effective in reducing IC in children with severe CP (Waller et al., 2013), although there is some suggestion that children with high levels of IC may need individualized and intensive interventions to produce sustained reductions in these features over time (Hyde, Waller, \& Burt, 2014).

To date, few longitudinal studies have examined the extent to which IC in youth is associated with later adult psychopathy. Lynam, Caspi, Moffitt, Loeber, and Stouthamer-Loeber (2007) found that parent-reported psychopathic personality features assessed at age 13 predicted adult psychopathic features assessed at age 24 among a community sample of boys. However, these features were primarily related to the erratic lifestyle $(r=.28)$ and crimimality $(r=.33)$ aspects of psychopathy, as opposed to the interpersonal $(r=.19)$ and affective $(r=.15)$ dimensions (Lynam, Caspi, Moffitt, Loeber, $\&$ Stouthamer-Loeber, 2007). In addition, this study assessed adolescent psychopathic features using a broad array of characteristics (e.g. impulsivity, dyregulated mood, irresponsibility), making it unclear whether the associations observed were attributable to individual differences in IC. In a related study, Burke, Loeber, and Lahey (2007) used data collected on a small sample of clinic-refered boys (ages $7-12$ ) to examine the aggregate association between teacher-reported IC assessed annually until age 17 and psychopathic features assessed at ages 18 and 19. Results indicated that there was a significant interaction between IC and symptoms of conduct disorder (CD) in models predicting the interpersonal/affective and irresponsible/criminality features of adult psychopathy. Specifically, IC was more strongly related adult psychopathy among adolescents with lower $\mathrm{CD}$ symptoms, and $\mathrm{CD}$ symptoms were more strongly related to adult psychopathy among boys with lower levels of IC features (also see Fanti, 2013).

Taken together, these longitudinal studies suggest that early IC may be a general risk marker for the development of a broader antisocial phenotype, rather than being a specific precursor to the affective/interpersonal features of adult psychopathy. This is consistent with a large number of studies demonstrating that IC in childhood and adolescence is a robust predictor of later adult violence and substance use (Baskin-Sommers, Waller, Fish, \& Hyde, 2015), arrests and convictions (Lynam, Miller, Vachon, Loeber, \& Stouthamer-Loeber, 2009), and antisocial personality features (Pardini \& Loeber, 2008). However, given that Burke et al. (2007) examined the association between early IC and later psychopathy within a clinic-referred sample of boys with high rates of $\mathrm{CP}$, the findings from this study may not be generalizable to community samples of youth.

Although existing longitudinal studies have exclusively examined early IC as a static risk factor for adult psychopathy, evidence suggests that substantive changes in IC do occur from childhood into adolescence (Byrd, Hawes, Loeber, \& Pardini, 2016; Pardini \& Loeber, 2008). Along these lines, there is some evidence that a significant portion of children experience significant reductions in IC over time (Byrd et al., 2016), and it is possible that this systematic decrease may reduce the likelihood that they will exhibit psychopathic features in adulthood. However, it is also possible that children with severe IC may continue to exhibit high levels of these features relative to their peers, even when they exhibit significant declines in IC over time (i.e. strong rank-order stability). In this instance, one would expect that children's early levels of IC would likely be a more robust predictor of adult psychopathy than changes in these features from childhood to adolescence. Importantly, no study has examined whether within-individual changes in IC during childhood are prospectively linked to psychopathy in adulthood. Such analyses are vital for garnering a clearer picture of the developmental course of psychopathic features over time (Roberts \& DelVecchio, 2000).

\section{Current study}

This study was designed to address several key issues regarding the association between IC and CP in youth and adult psychopathic traits. Using latent growth curve modeling, analyses examined the extent to which youth experienced significant within-individual change in both IC and CP during the transition from late childhood to early adolescence. Next, analyses examined whether initial levels and changes IC and CP during this developmental 
period were associated with the interpersonal/affective and lifestyle/criminality features of psychopathy assessed in early adulthood. Lastly, analyses examined whether there was an interaction effect between childhood levels IC and CP in predicting adult psychopathic traits.

\section{Method Design and participants}

Data from this study were collected as part of the Pittsburgh Youth Study (PYS), a longitudinal investigation aimed at understanding the development of delinquency, substance use, and mental health problems in at-risk boys (Loeber, Farrington, Stouthamer-Loeber, \& Van Kammen, 1998). The PYS consists of three cohorts of boys initially recruited from the Pittsburgh Public Schools in the 1st, 4th, and 7th grades. This study focuses on the middle PYS cohort, those youth who were recruited in the 4th grade, as follow-up for this cohort included an assessment of psychopathy in adulthood using the Psychopathy Checklist: Screening Version (PCL:SV; Hart, Cox, \& Hare, 1995). Boys were selected for the study following a multiinformant screening (parent, teacher, self-report) designed to assess early conduct problems (e.g. fighting, stealing). The screening was conducted on a random sample of 868 boys enrolled in the 4th grade. Boys who scored in the upper 30th percentile on the screener were selected for longitudinal followup $(N=259)$, along with a roughly equal number of boys randomly selected from those scoring below the 70 th percentile on the screener $(N=249)$. This led to a total sample of 508 boys. The racial/ethnic composition of the sample was as follows: Caucasian $=42.7 \%$, African-American $=52.4 \%$, and Other $=4.8 \%$.

\section{Procedures}

Data were collected across six biannual assessments, occuring at 6-month intervals beginning when the boys were 10-yearsold $(M=10.25, S D=0.79)$ and ending when the boys were 13 years-old $(M=13.27, S D=0.78)$. The study participants were then re-assessed in early adulthood approximately 11 years later $(M=24.19, S D=1.03)$ at which time they completed an semistructured interview to assess their level of psychopathic features. All procedures were reviewed and approved by the Institutional Review Board at the University of Pittsburgh. Informed consent was obtained prior to each assessment from participants or participant's parent/guardian. For more details about procedures, see Loeber et al. (1998).

\section{Measures}

Early interpersonal callousness: Interpersonal callousness (IC) was assessed using a previously validated scale (IC Scale; Pardini, Obradovic, \& Loeber, 2006) consisting of eight items from the Child Behavior Checklist (CBCL; Achenbach, 1991), or for teacher informants, the Teacher Report Form (TRF; Achenbach \& Edelbrock, 1986). Parents and teachers reported on the same eight items ('Exaggerates'; 'You can't trust what he says'; 'Denies wrongdoing'; 'Manipulates people'; 'Smooth talker, when confronted'; 'Acts sneakily'; 'Does not keep promises'; 'Not guilty after misbehaving'). Items were rated on a 3-point scale from 0 (not true) to 2 (very true) and combined across parent and teacher reports, taking the higher of the two ratings and summing them to create a total score (Byrd, Loeber, \& Pardini, 2012; Pardini et al., 2006). Unlike measures intended to tap into a broader 'callous-unemotional' construct, this measure of IC was does not provide a direct assessment of affective features such as 'unemotionality' and 'lack of empathy'. The IC scale has previously demonstrated evidence of a well-fitting factor structure and longitudinal invariance (Obradović, Pardini, Long, \& Loeber, 2007). The internal consistency was high across each assessment wave $(\alpha$ range $=.89-.92)$.

Conduct problems: The Self-Report of Delinquency (SRD; Elliott, Huizinga, \& Ageton, 1985) was used to measure conduct problems (CP). Participants were asked the number of times they had engaged in 32 different acts within the 'past 6 months' (i.e. the timespan since the previous assessment). A variety variable was created by summing the number of acts endorsed, including 11 items related to theft (e.g. stealing something $\$ 100+), 6$ items assessing violence (e.g. hitting someone with the intention of hurting them), and 16 items focused on other forms of delinquency (e.g. vandalism, drug selling). Across assessments, the mean number of different delinquent acts ranged from 1.30 to 1.56 (range $=0-20$ ).

Adult psychopathy: Psychopathy was assessed using the Psychopathy Checklist: Screening Version (PCL:SV; Hart et al., 1995), a 12-item abbreviated version of the 20-item Psychopathy Checklist- Revised (PCL-R; Hare, 2003). Items of the PCL:SV are rated from 0 to 2 (does not apply-applies), and consistent with two-factor models of the PCL- $R,{ }^{2}$ divide psychopathy into an affective/interpersonal component (i.e. factor 1) and an antisocial/lifestyle component (i.e. factor 2). To avoid potential overlap between study predictors and outcomes, a single PCL:SV item (adolescent antisocial behavior) was removed. In the current study, PCL:SV total and factor scores were modeled as latent constructs.

To score this measure, each participant was administered a semistructured interview lasting 30-45 min. Interviewers were college graduates with multiple years of experience and each completed training focused on PCL:SV administration and scoring. In addition, interviewers were blind to other study variables, and had no access to previous reports of psychopathy or offending. Interrater reliability, examined using intraclass correlations based on a single rater and absolute agreement, was good (total score ICC $=.86$ ), as was internal consistency (total score, $\alpha=.87$; factor $1, \alpha=.79$; factor 2 , $\alpha=.85$ ). For additional details regarding these procedures, see (Lynam et al., 2007).

\section{Data analytic plan}

Latent growth curve modeling (LGCM) was used to identify IC and $\mathrm{CP}$ trajectories. First, analyses examined the shape and model fit for each of these trajectories. Next, associations between the intercept and slope of each trajectory and adult psychopathy total and factor scores were examined. After investigating trajectories of IC and CP as separate predictors of adult psychopathy, we specified a bivariate LGCM, allowing for simultaneous examination of both trajectories within a single model and their unique associations with adult psychopathy, while controlling for their overlap. Finally, an interaction between the latent intercept factors of IC and CP predicting adult psychopapthy was examined. For all analyses, we examined prediction of adult psychopathy total, factor 1 , and factor 2 scores.

All models used maximum likelihood estimation with standard errors and a chi-square statistic that are robust to nonnormality (MLR) in Mplus 7.0 (Muthén \& Muthén, 19982012). Model fit was assessed using the Comparative Fit Index (CFI; Hu \& Bentler, 1999), Root Mean Square Error of Approximation (RMSEA; Browne, Cudeck, Bollen, \& Long, 1993), sample-size adjusted Bayesian Information Criterion (BIC), and the Akaike Information Criterion (AIC). Due to the 
count nature of the $\mathrm{CP}$ measure, a negative binomial link function was specified for this trajectory. The negative binomial model takes into consideration the over-dispersion of the count variable in the calculation of standard errors (Raftery, 1995), and provided an improvement in model fit compared to models specifying a continuous or Poisson distribution. The interaction between the latent intercepts was estimated using the latent moderated structural equation (LMS) method (Klein \& Moosbrugger, 2000) via the XWITH command and numerical integration in Mplus. Specifying interactions between latent variables has important benefits over more traditional approaches (e.g. ordinary least squares regression), as latent interactions are free of measurement error resulting in less loss of power or biased estimates (Busemeyer \& Jones, 1983; Kenny \& Judd, 1984; Little, Bovaird, \& Widaman, 2006).

\section{Missing data}

Throughout the course of the PYS, participant retention was high. The proportion of participants with complete data across childhood assessments in the current sample ranged from $94 \%$ to $99 \%$ across all six time points. Adult psychopathy data were available for 311 study participants. Full-information maximum likelihood (FIML) estimates were used to handle missing data, as this procedure uses all available data points to construct parameter estimates under the assumption that the data are missing at random. Therefore, the effective sample size for all study analyses was 507 (a single participant was missing data at each time point and thus not included in any analysis). There were no differences on initial risk scores, psychopathy or serious delinquency assessed at age 13, or SES status between participants having complete data and those missing data on the adulthood assessment (for additional details, see Lynam et al., 2007). Even when data are not missing at random, FIML estimation tends to produce less biased estimates than more traditional techniques for handling missing data (Enders \& Bandalos, 2001).

\section{Results \\ IC and CP growth trajectories}

First, model fit statistics and parameter estimates for both of the unconditional LGCMs were examined (Table 1). For the IC trajectory, there was no evidence of systematic mean-level change. However, findings did reveal significant variability in the rates of within-individual change in IC. This indicates that although the average IC trajectory for the sample appeared relatively stable, there were significant differences in patterns of change in IC across individuals from ages $10-13$ (slope- $b=-0.04, S E=.07$; range $=-2.5$ to 2.9 ). In contrast to $\mathrm{IC}$, there was a small, but systematic decrease in mean levels of $\mathrm{CP}$ over time. However, there was also significant variability in the rates of within-individual change in conduct problems between youth in the sample (slope- $\quad b=-0.11, S E=-.04 ; \quad$ range $=-0.72$ to 0.60).

\section{IC and CP trajectories predicting adult psychopathy}

Table 2 presents findings from analyses looking at the intercept and slope of IC and CP predicting adult psychopathy in separate models. Both the intercept (i.e. initial level) and slope (i.e. change over time) of IC significantly predicted adult psychopathy total scores, as well as factor 2 scores. The magnitude of these effects was moderate-tolarge, with the intercept demonstrating larger effects than the latent slope. The intercept factor of the IC trajectory, but not the slope factor, also predicted adult psychopathy factor 1 scores, with a moderate effect size. Nearly identical associations with adult psychopathy were demonstrated for the CP trajectory. However, in contrast to the IC model, the slope of $\mathrm{CP}$ also predicted adult factor 1 psychopathy scores, with a small-moderate effect size.

IC and CP trajectories were next combined into a parallel process growth model to assess their unique associations with adult psychopathy, while controlling for their overlap (see Table 3). An initial examination of this model showed moderate positive associations between the intercepts $(r=.40$, $p<.001$ ) and a small positive association between the slopes $(r=.24, p=.01)$ of these two trajectories. Findings from these analyses revealed that when controlling for the effect of the $\mathrm{CP}$, initial IC continued to significantly predict psychopathy total, factor 1 , and factor 2 scores. However, the magnitude of the effect sizes was attenuated after controlling for $\mathrm{CP}$, falling to the small-to-moderate range. In addition, the IC slope factor no longer predicted any adult psychopathy outcomes. Similar to the IC trajectory, the $\mathrm{CP}$ intercept continued to predict psychopathy total, factor 1 , and factor 2 scores. Unlike the IC model, change across development in CP (i.e. slope factor) remained a significant predictor of the psychopathy total and factor 2 scores in adulthood, even after controlling for the effects of IC. However, the CP

Table 1 Unconditional interpersonal callousness and conduct problems growth models

\begin{tabular}{|c|c|c|c|c|c|c|c|c|c|c|c|}
\hline & $\chi^{2}$ & $d f$ & CFI & RMSEA & AIC & ${ }_{\Delta} \mathrm{BIC}$ & Intercept $\bar{x}$ & Slope $\bar{x}$ & Intercept $\sigma^{2}$ & Slope $\sigma^{2}$ & $\operatorname{Cov}(\mathrm{I} / \mathrm{S})$ \\
\hline IC & 63.48 & 16 & .945 & .077 & 15,805 & 15,817 & $5.16^{* *}$ & -0.04 & $11.42^{* *}$ & $1.32^{* *}$ & $-1.68 * *$ \\
\hline $\mathrm{CP}$ & & & & & 8,072 & 8,084 & $-0.25^{* *}$ & $-0.11^{*}$ & $1.12^{* *}$ & $0.17^{* *}$ & -0.05 \\
\hline
\end{tabular}

Estimates are presented in unstandardized form. The conduct problems model was specified using a negative binomial function (type $=$ random). Fit indices and standardized estimates are unavailable for this model.

${ }_{\Delta} \mathrm{BIC}=$ Sample-Size Adjusted BIC.

Cov $(\mathrm{I} / \mathrm{S})=$ Covariance of the model intercept and slope parameters.

${ }^{*} p<.05 ;{ }^{* *} p<.01$. 
slope factor was not related to factor 1 psychopathy scores in adulthood when controlling for IC intercept and slope factors.

\section{Latent trajectory interaction}

Finally, we tested for an interaction between the latent intercepts of the IC and $\mathrm{CP}$ trajectories in predicting each of the adult psychopathy outcomes. ${ }^{3}$ This latent interaction analysis revealed a significant two-way interaction between intercept factors predicting adult psychopathy total $(\mathrm{B}=0.03, S E=.01$, $p=.03)$ and factor $2(\mathrm{~B}=0.04, S E=.02, p=.04)$ scores but not factor 1 scores (Table 3; Figure S1, available online). Probing the significant interaction revealed that boys were at the greatest risk of demonstrating adulthood psychopathy if they exhibited high levels of both IC and CP in late childhood (Figures 1A,B and 2). The inclusion of the latent interaction term significantly improved the model. In the prediction of total psychopathy score, the overall model $R^{2}$ without the interaction term was .17, whereas inclusion of the interaction increased this value to $.21\left(\Delta R^{2}=.04\right)$. Similarly, the model predicting factor 2 produced an $R^{2}$ of .24 , which increased to .26 after including the interaction term $\left(\Delta R^{2}=.02\right)$.

\section{Discussion}

This study highlights several novel findings. First, evidence of important individual differences in the developmental course of both IC and CP were identified. These findings are consistent with, but expand on prior research in this area (e.g. Baskin-Sommers et al., 2015; Fontaine, McCrory, Boivin, Moffitt, \& Viding, 2011; Pardini \& Loeber, 2008), by providing evidence of within-individual change in these constructs during the transition to adolescence. Our investigation into the long-term prospective prediction of these early manifestations of IC and CP revealed that each of these constructs exhibited important associations with adult psychopathy.

Table 2 Interpersonal callousness and conduct problems growth trajectories predicting adult psychopathy scores in separate models

\begin{tabular}{|c|c|c|c|}
\hline & PCL total & PCL factor 1 & PCL factor 2 \\
\hline \multicolumn{4}{|c|}{ Interpersonal callousness trajectory } \\
\hline $\begin{array}{l}\text { Intercept } \\
\text { ( age 10) }\end{array}$ & $0.50(.06)^{* * *}$ & $0.33(.07)^{* * *}$ & $0.56(.06)^{* * *}$ \\
\hline $\begin{array}{l}\text { Linear slope } \\
\text { ( age 10-13) }\end{array}$ & $0.29(.10)^{* *}$ & $0.25(.13)$ & $0.30(.10)^{* * *}$ \\
\hline \multicolumn{4}{|c|}{ Conduct problems trajectory } \\
\hline $\begin{array}{l}\text { Intercept } \\
\text { ( age 10) }\end{array}$ & $0.47(.06)^{* * *}$ & $0.32(.06)^{* * *}$ & $0.51(.06)^{* * *}$ \\
\hline $\begin{array}{l}\text { Linear slope } \\
\text { ( age 10-13) }\end{array}$ & $0.26(.08)^{* *}$ & $0.21(.07)^{* *}$ & $0.28(.07)^{* * *}$ \\
\hline
\end{tabular}

$* p<.05 ; * * p<.01 ; * * * p<.001$. All reported parameter estimates are standardized.
Table 3 Parallel process growth model examining interpersonal callousness and conduct problems trajectories as unique predictors of adult psychopathy

\begin{tabular}{|c|c|c|c|}
\hline & PCL total & PCL factor 1 & PCL factor 2 \\
\hline \multicolumn{4}{|l|}{ Main effects } \\
\hline \multicolumn{4}{|c|}{ Interpersonal callousness trajectory } \\
\hline $\begin{array}{l}\text { Intercept } \\
\text { ( age 10) }\end{array}$ & $0.29(.07)^{* * *}$ & $0.18(.08)^{*}$ & $0.29(.08)^{* * *}$ \\
\hline $\begin{array}{l}\text { Linear slope } \\
\text { ( age 10-13) }\end{array}$ & $0.15(.10)$ & $0.16(.14)$ & $0.16(.10)$ \\
\hline \multicolumn{4}{|c|}{ Conduct problems trajectory } \\
\hline $\begin{array}{l}\text { Intercept } \\
\text { ( age 10) }\end{array}$ & $0.34(.07)^{* * *}$ & $0.23(.07)^{* *}$ & $0.40(.07)^{* * *}$ \\
\hline $\begin{array}{l}\text { Linear slope } \\
\text { ( age 10-13) }\end{array}$ & $0.18(.08)^{*}$ & $0.14(.08)$ & $0.21(.08)^{*}$ \\
\hline \multicolumn{4}{|l|}{ Interaction } \\
\hline $\begin{array}{l}\text { Interpersonal } \\
\text { callousness } \times \\
\text { Conduct } \\
\text { problems } \\
\text { intercepts }\end{array}$ & $0.03(.01)^{*}$ & $0.05(.04)$ & $0.04(.02)^{*}$ \\
\hline
\end{tabular}

${ }^{*} p<.05 ;{ }^{* *} p<.01 ;{ }^{* * *} p<.001$. Standardized parameter estimates are reported for all main effects. Interaction effects are reported in unstandardized units, as standardized estimates are generally not available for interactions between latent variables.

Notably, results from this study revealed an interaction between initial levels of IC and CP. Specifically, boys with the highest initial levels of both IC and CP in late childhood were at greatest risk for psychopathy in early adulthood. Although this is consistent with theory and research suggesting that this subgroup of youth is at heightened risk for the development of psychopathy (Frick \& White, 2008), this is the first study to prospectively demonstrate that these features and their interaction predict adult psychopathy.

Significant heterogeneity between-individuals was found for both initial levels and rates of change in both IC and CP from late childhood into early adolescence. Importantly, results demonstrate that youth with high levels of IC or CP in late childhood are not on an enduring or fixed course for developing adult psychopathy (see Edens, Marcus, Lilienfeld, \& Poythress, 2006). In addition, the demonstrated heterogeneity in the rates of change in features of IC and CP across development, which may be masked in studies examining between-individual differences at a single point in time, highlights the importance of research using prospective longitudinal designs.

Importantly, although there is support for the notion that IC is not an immutable facet of personality, boys with the highest levels of IC or CP in late childhood were at heightened risk for exhibiting psychopathic features in early adulthood. Across both univariate and bivariate analyses, results consistently demonstrated the value of IC and CP as predictors of total psychopathy scores. Findings appeared stronger for the lifestyle/antisocial factor (i.e. factor 2), which is consistent with previous work 

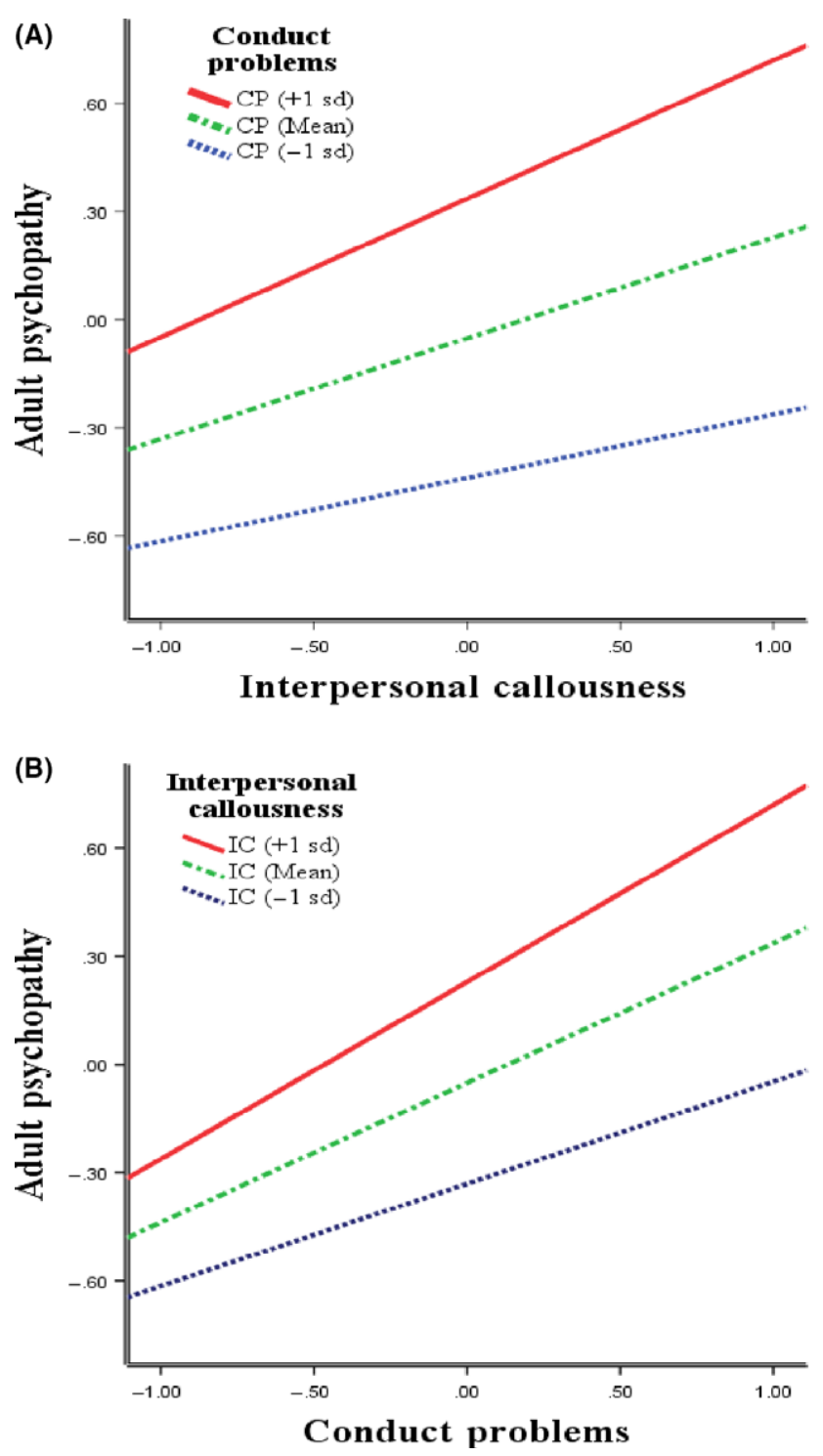

Figure 1 (A) Early CP moderating early IC and adult psychopathy. (B) Early IC moderating early CP and adult psychopathy. To aid in graphical interpretation, study variables were standardized to scale each into standard deviation units

in this area showing robust and unique influences on antisocial behavior as opposed to the interpersonal/affective characteristics (Burke et al., 2007). This may point to the interpersonal/affective features of adult psychopathy being more difficult to predict in the longer term, as compared to lifestyle/ antisocial features. However, this could also reflect that the IC construct, which lacks items assessing characteristics such as 'shallow emotions' or 'lack of empathy', does not map onto the interpersonal/ affective dimension of the PCL measure, as well as the CP construct does with the lifestyle/antisocial factor.

Although initial levels of IC were associated with both factors of adult psychopathy, change in IC from late childhood to early adolescence was only predictive of the lifestyle/antisocial component of adult psychopathy. Several factors may influence the noted lack association between change in IC across

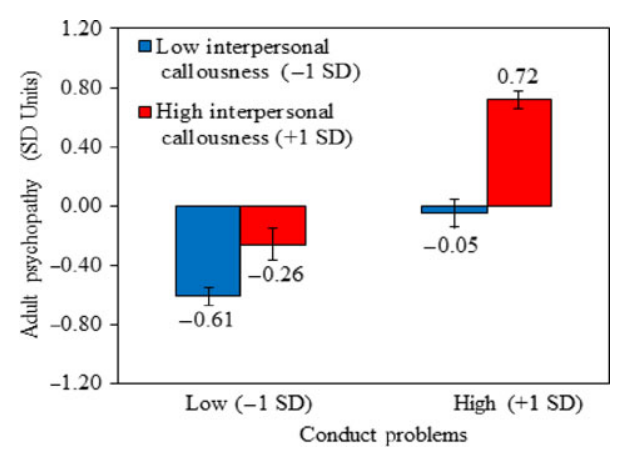

Figure 2 Bar graph of early IC moderating early CP and adult psychopathy

the study period and the interpersonal/affective features of adult psychopathy. It may be that these results simply indicate that initial levels of IC already explain the lionshare of the relationship between early IC and the interpersonal/affective features of adult psychopathy. Similarly, the lack of a significant association between changes in $\mathrm{CP}$ and the interpersonal/affective component of adult psychopathy in the full parallel process growth model may be largely due to the amount variance explained when accounting for initial levels of each of these constructs. Along these lines, findings may reflect that there are different developmental periods wherein rates of change in early IC and CP exert differing amounts of influence on adult psychopathy. For example, some research suggests that change in IC during earlier childhood years may be particularly important (e.g. Frick et al., 2003), whereas a large body of literature points toward the influence of pubertal based changes on CP. Alternatively, this finding may reflect the need to derive more developmentally driven and bottom-up approaches for assessing IC, rather than relying on adapting or downwardly extending items to children that were originally designed to tap the construct in adults.

Our findings highlight that boys who exhibit high levels of both IC and CP, even as early as 10 years of age, are at heightened risk for exhibiting later psychopathic features. Intervention research suggests that targeting multiple domains of risk, particularly in younger youth, may lead to improvements in IC over time, with treatments facilitating improvements in parenting practices, child social skills, and child problem solving leading to reductions in IC (for a review see Wilkinson, Waller, \& Viding, 2015). In addition to early prevention and intervention efforts that have shown promising effects for youth with high IC (Hawes, Price, \& Dadds, 2014; Waller et al., 2013), clinical pessimism about the absolute stability of these characteristics should be tempered in light of documented variability in these features over time.

\section{Limitations}

This study was characterized by a number of strengths including a prospective longitudinal design 
spanning over 15 years, inclusion of a large racially diverse sample, and use of multiple informants. However, there are several noteworthy limitations. First, this study focused on a community sample of atrisk males, limiting generalizability to females or clinical populations. Second, assessments of IC and $\mathrm{CP}$ were conducted during late childhood and early adolescence. A number developmental processes relevant to IC and CP are thought to take place during earlier childhood periods (Hawes, Price, et al., 2014; Wilkinson et al., 2015). Indeed, 10-years-old is the recommended cut-off for designating childhood-onset $\mathrm{CD}$. Consequently, future studies examining these associations beginning during earlier periods of development are critical. Third, the measurement of IC is not directly comparable to more traditional measures of callous-unemotional traits, such as the CU traits scale from the Antisocial Processes Screening Device (Frick \& Hare, 2001) or the Inventory of Callous Unemotional Traits (Frick, 2004). Moreover, items indexing IC were obtained post hoc from archival data, and none of the items specifically assessed affective features concerning a lack of empathy or shallow affect. Replication of these results using instruments designed to provide a more direct assessment of CU traits is an important avenue for future studies. Nevertheless, the IC construct has been validated using all three cohorts of the PYS (Pardini et al., 2006) and has demonstrated longitudinal invariance across childhood and adolescence (Obradović et al., 2007).

\section{Summary}

This study broadens our understanding of the development of IC and CP from late childhood to early adolescence and risk for psychopathy in early adulthood. Findings revealed evidence of significant within-person change in trajectories of IC and CP and that these trajectories provided independent and unique prediction of adult psychopathy. Importantly, there was a significant interaction between initial levels of IC and CP, whereby youth highest on both were at greatest risk for exhibiting psychopathic features as adults. Taken together, results highlight individual variability in the developmental course of IC and CP and point to the importance of directing intervention efforts toward youth demonstrating elevated IC and CP in late childhood.

\section{Supporting information}

Additional Supporting Information may be found in the online version of this article:

Appendix S1. Item descriptors for the Interpersonal Callousness (IC) Scale, Inventory of Callous-Unemotional Traits (ICU), and Antisocial Process Screen Device (APSD).

Figure S1. Parallel process latent growth trajectories and interaction effect predicting adult psychopathy.

\section{Acknowledgements}

This research was supported in part by NIMH grants MH45070, MH49414 and MH60104; grant 86-JNCX0009 from the Office of Juvenile Justice and Delinquency, Office of Justice Programs, and the U.S. Department of Justice. The authors have declared that they have no competing or potential conflicts of interests.

\section{Correspondence}

Samuel W. Hawes, Department of Psychiatry, University of Pittsburgh Medical Center, 201 N. Craig Street, Pittsburgh, PA, USA; Email: Samuel.hawes@asu.edu

\section{Key points}

- Interpersonal callousness and conduct problems tend to co-occur in youth.

- Substantive changes in interpersonal callousness and conduct problems occur for a subset of boys from late childhood to early adolescence.

- Boys exhibiting a combination of high interpersonal callousness and conduct problems during late childhood are at particularly high risk for developing adult psychopathic features.

- Boys who exhibit significant reductions in interpersonal callousness and conduct problems during the transition to adolescence are less likely to develop adult psychopathic features.

- Intensive empirically based interventions for boys exhibiting high interpersonal callousness and conduct problems may help prevent the development of adult psychopathy.

\section{Notes}

1. Similar to other common measures of callousunemotional traits, including the APSD and ICU, the IC scale assesses manipulativeness, uncaring for others, lack of concern about punishment. Unlike the APSD and ICU, however, the IC scale does not include items tapping lack of empathy or shallow emotions (see Supplemental Appendix 1, available online). As such, the IC measure contains fewer items intended to directly assess affective features, comparative to these other measures. Thus, although the IC construct shares some overlap with measures of callous-unemotional traits (e.g. 
callousness), these constructs are not synonymous, and there are notable differences (e.g. IC does not directly assess affective features such as lack of emotionality', 'lack of empathy').

2. There is ongoing debate in the literature that is beyond the scope of the current paper centers on the utility of this two-factor approach versus a three- or four-factor/facet approach (for a discussion of this debate see Skeem, Polaschek, Patrick, \& Lilienfeld, 2011).

3. Although the slope factor of the callousness trajectory did not significantly predict any outcome when accounting for the influence of the conduct problems trajectory, analyses were conducted to investigate possible interaction effects between slope factors. Findings demonstrated no relationship between the latent slope interaction and any adult outcome variable. Results provided upon request.

\section{References}

Achenbach, T.M. (1991). Manual for the Child Behavior Checklist/4-18 and 1991 profiles. Burlington, VT: University of Vermont, Department of Psychiatry.

Achenbach, T.M., \& Edelbrock, C. (1986). Manual for the teacher's report form and teacher version of the child behavior profile. Burlington, VT: University of Vermont, Department of Psychiatry.

Baskin-Sommers, A.R., Waller, R., Fish, A.M., \& Hyde, L.W. (2015). Callous-unemotional traits trajectories interact with earlier conduct problems and executive control to predict violence and substance use among high risk male adolescents. Journal of Abnormal Child Psychology, 43, 15291541.

Browne, M.W., Cudeck, R., Bollen, K.A., \& Long, J.S. (1993). Alternative ways of assessing model fit. Sage Focus Editions, 154, 136-162.

Burke, J.D., Loeber, R., \& Lahey, B.B. (2007). Adolescent conduct disorder and interpersonal callousness as predictors of psychopathy in young adults. Journal of Clinical Child and Adolescent Psychology, 36, 334-346.

Busemeyer, J.R., \& Jones, L.E. (1983). Analysis of multiplicative combination rules when the causal variables are measured with error. Psychological Bulletin, 93, 549.

Byrd, A.L., Hawes, S.W., Loeber, R., \& Pardini, D.A. (2016). Interpersonal callousness from childhood to adolescence: Developmental trajectories and early risk factors. Journal of Clinical Child \& Adolescent Psychology. Advanced online publication. doi:10.1080/15374416.2016.1144190.

Byrd, A.L., Loeber, R., \& Pardini, D.A. (2012). Understanding desisting and persisting forms of delinquency: The unique contributions of disruptive behavior disorders and interpersonal callousness. Journal of Child Psychology and Psychiatry, 53, 371-380.

Edens, J.F., Marcus, D.K., Lilienfeld, S.O., \& Poythress Jr, N.G. (2006). Psychopathic, not psychopath: Taxometric evidence for the dimensional structure of psychopathy. Journal of Abnormal Psychology, 115, 131.

Elliott, D.Z., Huizinga, D., \& Ageton, S.S. (1985). Explaining delinquency and drug use. Beverly Hills, CA: Sage.

Enders, C.K., \& Bandalos, D.L. (2001). The relative performance of full information maximum likelihood estimation for missing data in structural equation models. Structural Equation Modeling, 8, 430-457.

Fanti, K.A. (2013). Individual, social, and behavioral factors associated with co-occurring conduct problems and callous- unemotional traits. Journal of Abnormal Child Psychology, $41,811-824$.

Fontaine, N.M., McCrory, E.J., Boivin, M., Moffitt, T.E., \& Viding, E. (2011). Predictors and outcomes of joint trajectories of callous-unemotional traits and conduct problems in childhood. Journal of Abnormal Psychology, 120, 730.

Frick, P.J. (2004). The inventory of callous-unemotional traits. Unpublished rating scale.

Frick, P.J., Cornell, A.H., Bodin, S.D., Dane, H.E., Barry, C.T., \& Loney, B.R. (2003). Callous-unemotional traits and developmental pathways to severe conduct problems. Developmental Psychology, 39, 246-260.

Frick, P.J., \& Hare, R.D. (2001). Antisocial process screening device technical manual. Toronto, ON: Multi-Health Systems.

Frick, P.J., \& White, S.F. (2008). Research review: The importance of callous-unemotional traits for developmental models of aggressive and antisocial behavior. Journal of Child Psychology and Psychiatry, 49, 359-375.

Hare, R.D. (2003). Hare psychopathy checklist-revised (PCL-R) (2nd edn). North Toawanda, NY: Multi-Health Systems.

Hart, S.D., Cox, D.N., \& Hare, R.D. (1995). Manual for the psychopathy checklist: Screening version (PCL:SV). Toronto, ON: Multi-Health Systems.

Hawes, S.W., Mulvey, E.P., Schubert, C.A., \& Pardini, D.A. (2014). Structural coherence and temporal stability of psychopathic personality features during emerging adulthood. Journal of Abnormal Psychology, 123, 623.

Hawes, D.J., Price, M.J., \& Dadds, M.R. (2014). Callousunemotional traits and the treatment of conduct problems in childhood and adolescence: A comprehensive review. Clinical Child and Family Psychology Review, 17, 248-267.

Hu, L., \& Bentler, P.M. (1999). Cutoff criteria for fit indexes in covariance structure analysis: Conventional criteria versus new alternatives. Structural Equation Modeling, 6, $1-55$.

Hyde, L.W., Shaw, D.S., Gardner, F., Cheong, J., Dishion, T.J., \& Wilson, M. (2013). Dimensions of callousness in early childhood: Links to problem behavior and family intervention effectiveness. Development and Psychopathology, 25, 347-363.

Hyde, L.W., Waller, R., \& Burt, S.A. (2014). Commentary: Improving treatment for youth with callous unemotional traits through the intersection of basic and applied science reflections on Dadds et al. (2014). Journal of Child Psychology and Psychiatry, 55, 781-783.

Kenny, D.A., \& Judd, C.M. (1984). Estimating the nonlinear and interactive effects of latent variables. Psychological Bulletin, 96, 201.

Klein, A., \& Moosbrugger, H. (2000). Maximum likelihood estimation of latent interaction effects with the LMS method. Psychometrika, 65, 457-474.

Little, T.D., Bovaird, J.A., \& Widaman, K.F. (2006). On the merits of orthogonalizing powered and product terms: Implications for modeling interactions among latent variables. Structural Equation Modeling, 13, 497-519.

Loeber, R., Farrington, D.P., Stouthamer-Loeber, M., \& Van Kammen, W.B. (1998). Antisocial behavior and mental health problems: Explanatory factors in childhood and adolescence. New York: Routledge.

Lynam, D.R., Caspi, A., Moffitt, T.E., Loeber, R., \& StouthamerLoeber, M. (2007). Longitudinal evidence that psychopathy scores in early adolescence predict adult psychopathy. Journal of Abnormal Psychology, 116, 155-165.

Lynam, D.R., Miller, D.J., Vachon, D., Loeber, R., \& Stouthamer-Loeber, M. (2009). Psychopathy in adolescence predicts official reports of offending in adulthood. Youth Violence and Juvenile Justice, 7, 189-207.

Muthén, L.K., \& Muthén, B.O. (1998-2012). Mplus user's guide (7th edn). Los Angeles: Muthén \& Muthén. 
Obradović, J., Pardini, D.A., Long, J.D., \& Loeber, R. (2007). Measuring interpersonal callousness in boys from childhood to adolescence: An examination of longitudinal invariance and temporal stability. Journal of Clinical Child and Adolescent Psychology, 36, 276-292.

Pardini, D.A., \& Loeber, R. (2008). Interpersonal callousness trajectories across adolescence: Early social influences and adult outcomes. Criminal Justice and Behavior, 35, 173196.

Pardini, D.A., Loeber, R., \& Stouthamer-Loeber, M. (2005). Developmental shifts in parent and peer influences on boys' beliefs about delinquent behavior. Journal of Research on Adolescence, 15, 299-323.

Pardini, D.A., Obradovic, J., \& Loeber, R. (2006). Interpersonal callousness, hyperactivity/impulsivity, inattention, and conduct problems as precursors to delinquency persistence in boys: A comparison of three grade-based cohorts. Journal of Clinical Child and Adolescent Psychology, 35, 46-59.

Pardini, D.A., Waller, R., \& Hawes, S.W. (2015). Familial influences on the development of serious conduct problems and delinquency. In: J. Morizot \& L. Kazemian (Eds.), The development of criminal and antisocial behavior (pp. 201-220). Dordrecht: Springer International.

Patrick, C.J. (2007). Getting to the heart of psychopathy. Mahwah, NJ: Lawrence Erlbaum.

Raftery, A.E. (1995). Bayesian model selection in social research. Sociological Methodology, 25, 111-164.
Rice, M.E., \& Harris, G.T. (1997). The treatment of mentally disordered offenders. Psychology, Public Policy, and Law, 3, $126-183$.

Roberts, B.W., \& DelVecchio, W.F. (2000). The rank-order consistency of personality traits from childhood to old age: A quantitative review of longitudinal studies. Psychological Bulletin, 126, 3-25.

Seagrave, D., \& Grisso, T. (2002). Adolescent development and the measurement of juvenile psychopathy. Law and Human Behavior, 26, 219-239.

Skeem, J.L., \& Cooke, D.J. (2010). Is criminal behavior a central component of psychopathy? Conceptual directions for resolving the debate. Psychological Assessment, 22, 433-445.

Skeem, J.L., Polaschek, D.L., Patrick, C.J., \& Lilienfeld, S.O. (2011). Psychopathic personality bridging the gap between scientific evidence and public policy. Psychological Science in the Public Interest, 12, 95-162.

Waller, R., Gardner, F., \& Hyde, L.W. (2013). What are the associations between parenting, callous-unemotional traits, and antisocial behavior in youth? A systematic review of evidence. Clinical Psychology Review, 33, 593-608.

Wilkinson, S., Waller, R., \& Viding, E. (2015). Practitioner review: Involving young people with callous unemotional traits in treatment-does it work? A systematic review. Journal of Child Psychology and Psychiatry, 57, 552-565.

Accepted for publication: 7 June 2016

First published online: 12 August 2016 